\title{
Updated Perspectives on the Management of Multiple Myeloma in Older Patients: Focus on Lenalidomide
}

This article was published in the following Dove Press journal:

Clinical Interventions in Aging

\section{Kelly L Schoenbeck' Tanya M Wildes $\mathbb{D}^{2}$}

'Department of Medicine, University of California San Francisco, San Francisco, CA, USA; ${ }^{2}$ Department of Medicine, Washington University School of Medicine, St Louis, MO, USA
Correspondence: Tanya M Wildes Department of Medicine, Washington University School of Medicine, 660 South Euclid Ave, Campus Box 8056, St Louis, MO 63110, USA

$\mathrm{Tel}+$ I 3|4-273-3073

Email twildes@wustl.edu

\begin{abstract}
Multiple myeloma is a hematologic malignancy that predominantly affects older adults, with a median age at diagnosis of 70 years old. A mainstay of multiple myeloma treatment is lenalidomide, which is an immunomodulatory drug (IMiD) that changed the treatment paradigm for multiple myeloma. This is particularly true for older adults who do not undergo autologous stem cell transplantation (ASCT). Several pivotal trials summarized in this review demonstrate the efficacy and safety of lenalidomide in older adults with multiple myeloma, including significant improvements in response rates, progression-free survival and overall survival in the first-line and relapsed/refractory settings. Potential adverse effects include venous thromboembolism, cytopenias, and second malignancies and the doses tolerated in real-world older patients are often lower than those utilized in clinical trials enrolling select older patients. Given the heterogeneity of aging, several approaches to measuring frailty have been developed and validated to aid in predicting which older adults may benefit from empiric dose reduction to reduce the risk of toxicity and improve the tolerability of treatment. A number of randomized trials have explored a range of approaches utilizing lenalidomide in older adults in both the up-front and relapsed setting, ranging from attenuated maintenance strategies through quadruplet combination therapies including proteasome inhibitors and monoclonal antibodies. This wealth of literature provides a great number of options, which can make it difficult for a clinician to determine a single optimal recommendation for an individual patient. While lenalidomide is currently part of standard of care, the treatment of multiple myeloma is growing rapidly. There is a need to expand clinical trials participation to older adults with multiple myeloma. Incorporation of validated comprehensive geriatric assessments in clinical trials for multiple myeloma could provide a more accurate depiction of the older patient population and is an area for future exploration.
\end{abstract}

Keywords: multiple myeloma, lenalidomide, older adults, clinical trials

\section{Introduction}

Multiple myeloma is an incurable hematologic malignancy characterized by the production of malignant plasma cells, leading to anemia, lytic bone lesions, renal dysfunction, and hypercalcemia. Multiple myeloma predominantly impacts older adults, with a median age at diagnosis of 70 years old, with approximately one-third of patients diagnosed when they are older than 75 years. ${ }^{1,2}$ Multiple myeloma comprises an estimated $12-15 \%$ of all hematologic malignancies, with an increasing incidence among older adults; the number of new myeloma cases in adults older than 65 years 
old is projected to double between 2010 and $2030 .^{1-3}$ Treatment advances during the last few decades have led to increases in overall survival. ${ }^{4}$ However, there is a notable difference in survival of multiple myeloma patients under the age of 65 years old compared to those over 75 , and those over 75 experience the highest rates of disease-related mortality. ${ }^{4-6}$ The survival differences are thought to be multifactorial, with medical comorbidities and functional status being important factors that impact treatment options and patient outcomes. ${ }^{5}$

One of the primary initial treatment decisions in multiple myeloma is determining whether patients are candidates for high-dose chemotherapy followed by autologous stem cell transplantation (ASCT). ASCT is a mainstay of multiple myeloma treatment in those younger than 65 years old, as randomized trials show improved overall survival (OS) and progression-free survival (PFS) compared to standard therapy., ${ }^{7,8}$ Since patients older than 65 years were not included in the pivotal trials establishing ASCT in myeloma, the role for ASCT in older patients is not definitively known, although retrospective analyses have shown its successful use in select older adults. ${ }^{9}$ While age is not an absolute contraindication to ASCT, older adults may have aging-associated vulnerabilities, such as medical comorbidities, poor functional status, cognitive impairment, or lack of psychosocial support, with each potentially increasing the risks associated with ASCT and decreasing the likelihood of its use. ${ }^{10}$ Ultimately, the decision to perform ASCT in an older adult is determined by the transplanting center and physician. Patients over 65 years comprised fewer than $20 \%$ of those who underwent ASCT for multiple myeloma between 2006 and 2010, ${ }^{11}$ although the use of ASCT in older patients has been increasing over time. In 2017, 28\% of ASCT were performed in older adults, with similar outcomes for patients who underwent ASCT at age 70 and older compared to those between the ages of $60-69 .{ }^{12}$ Despite the increasing use of ASCTs in older adults, they are still not being used in the majority of older multiple myeloma patients. In part that has to do with the median age at which patients are diagnosed with multiple myeloma. ${ }^{13}$ Given that most older patients with multiple myeloma do not undergo ASCT, other therapeutic options that are also associated with increased overall survival frequently become the focus of their treatment plan.

One such treatment option is lenalidomide, which is included in multiple regimens for both transplant-eligible and transplant-ineligible patients with multiple myeloma. Lenalidomide is an immunomodulatory drug (IMiD) that is a derivative of thalidomide. Thalidomide was first developed in the 1950s and was used to treat pregnancy-associated nausea. However, it was later found to cause significant congenital abnormalities and was denied FDA approval in the 1960s, and ultimately led the FDA to change its approval and monitoring practices. Further research on thalidomide found that it impacts the immune system and impairs angiogenesis, making it a promising treatment for malignancies. Thalidomide showed activity against multiple myeloma and was FDA approved for multiple myeloma in $2006 .{ }^{14}$ However, thalidomide has associated toxicities, including peripheral neuropathy, somnolence, and constipation. ${ }^{15}$ As a consequence, lenalidomide was developed as a less toxic, more potent IMiD. Lenalidomide's anti-myeloma effects are mediated through immunomodulation, anti-angiogenesis, and antineoplastic activity, which directly impact the myeloma cells as well as the microenvironment. ${ }^{15,16}$ Lenalidomide's significant activity in multiple myeloma has led to its incorporation into multiple treatment regimens, including several for older adults with multiple myeloma who do not undergo ASCT (Figure 1). As such, the primary focus of this review is on the trials (Table 2) demonstrating the utility of lenalidomide in older multiple myeloma patients who are not candidates for ASCT.

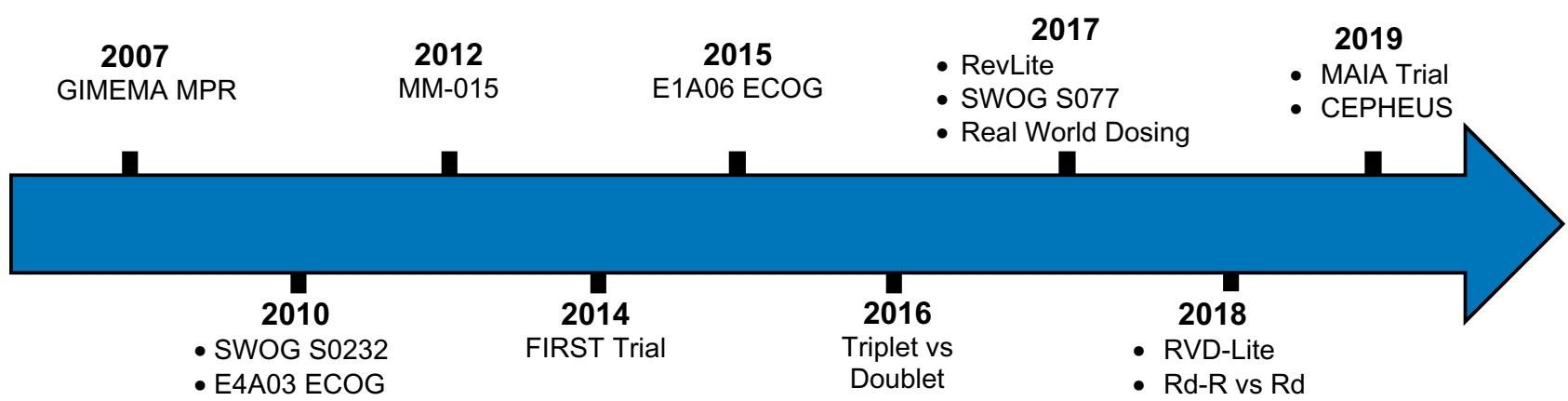

Figure I Timeline of important lenalidomide trials in the treatment of older adults with multiple myeloma. 


\section{Lenalidomide and Melphalan-Based Regimens GIMEMA Melphalan, Prednisone, Lenalidomide (MPR): First-Line Lenalidomide in Older Adults (2007)}

The GIMEMA - Italian Multiple Myeloma Network performed one of the first trials to examine the use of lenalidomide in the first-line setting in older patients with multiple myeloma. They assessed the combination of melphalan, prednisone, and lenalidomide in a Phase I/II dose-finding, comparative, open-label study. All patients were over 65 years old or ineligible for high-intensity treatment. Ultimately, 54 patients enrolled, with a median age of 71 . Melphalan was dosed between 0.18 and $0.25 \mathrm{mg} / \mathrm{kg}$ on days $1-4$, prednisone $2 \mathrm{mg} / \mathrm{kg}$ on days $1-4$, and lenalidomide 5-10 $\mathrm{mg}$ daily on days 1-21 out of a 28-day cycle for a total of 9 cycles. After completion of 9 cycles, participants were treated with lenalidomide maintenance alone. The study found a maximumtolerated dose of melphalan $0.18 \mathrm{mg} / \mathrm{kg}$ and lenalidomide $10 \mathrm{mg}$, with $23.8 \%$ participants achieving complete response (CR) and $47.6 \%$ achieving a very good partial response (VGPR). The overall survival (OS) at one-year was 100\% in all participants. Grade 3 toxicities included cytopenias, febrile neutropenia, and thromboembolism. This study showed the promise of using lenalidomide in the first-line setting for older patients with multiple myeloma and set the precedent for future trials. ${ }^{17}$

\section{Multiple Myeloma 0 I5 (MM-0I5) (20I2)}

While research continued evaluating the role of lenalidomide in the first-line setting for transplant-ineligible patients, lenalidomide was also showing improved PFS and time to progression as maintenance therapy after ASCT compared to placebo. ${ }^{18,19}$ Similarly, MM-015 sought to evaluate the role for post-induction maintenance lenalidomide in those who were ineligible for ASCT. MM015 was a Phase 3, randomized, double-blind, placebocontrolled trial for patients age 65 or older with a new diagnosis of multiple myeloma with three arms: melphalan, prednisone, and lenalidomide induction followed by lenalidomide maintenance (MPR-R); melphalan, prednisone, and lenalidomide (MPR) induction followed by placebo; melphalan and prednisone (MP) induction with placebo during both induction and maintenance periods. All treatment arms used the same dosing of melphalan and prednisone during induction, and MPR-R and MPR also included lenalidomide $10 \mathrm{mg}$ on days $1-21$ in a 28-day cycle for a total of 9 cycles. The MPR-R lenalidomide maintenance consisted of $10 \mathrm{mg}$ daily on days 1-21 until disease progression or intolerable adverse effects. A total of 459 patients were randomized to each arm with a median age of 71 . The arms were not well balanced for performance status as measured by Karnofsky Performance Status (KPS), with high average KPS in the MP group (90\%) compared to a KPS of $80 \%$ in the MPR$\mathrm{R}$ and MPR arms $(\mathrm{P}=0.03)$. Of note, either the KPS or the Eastern Cooperative Oncology Group (ECOG) scoring system is used to describe the performance status of oncology patients, with KPS scores ranging from 0 (dead) to 100 (fully functional) and ECOG scores from 5 (dead) to 0 (fully functional). Overall, MPR-R was found to have significantly longer PFS (31 months) compared to MPR (14 months; HR 0.49; $\mathrm{P}<0.001$ ) or MP (13 months; HR $0.4 ; \mathrm{P}<0.001)$. MPR-R showed particular benefit in those between the ages 65-75, as they had significantly longer PFS (31 months) than MPR (15 months; HR 0.48; $\mathrm{P}<$ 0.001 ) or MP (12 months; HR 0.3; P< 0.001). However, in those older than age 75 , the MPR-R PFS advantage was not nearly as striking; MPR-R had a PFS of 19 months compared to 12 months with MPR and 15 months with MP. PFS was associated with lenalidomide maintenance in all age groups in a landmark analysis, which showed lenalidomide maintenance reduced disease progression by $66 \%$ compared to placebo (MPR-R vs MPR HR 0.34; $\mathrm{P}<0.001)$. There was no significant difference in OS. Toxicities included cytopenias, which frequently occurred during induction in the MPR-R and MPR arms. Drug discontinuation due to adverse events occurred across all study arms, although more so in MPR-R (16\%) and MPR (14\%) than MP (5\%). At three years, there was an increased risk of second malignancies in the MPR-R and MPR arms (each 7\%) compared to the MP arm (3\%). ${ }^{20}$ Ultimately, given the improvement in PFS in older patients ineligible for ASCT, the MM-015 study helped establish the role of maintenance lenalidomide that was replicated in later trials.

\section{EIA06 ECOG Trial: MPT-T versus mPR-R (20I5)}

The E1A06 study sought to study the combination of lenalidomide with prednisone and low-dose melphalan (mPR) versus MPT using a non-inferiority design in older adults with new diagnoses of multiple myeloma. 
This was a phase 3 randomized controlled trial (RCT) for patients 65 or older or patients otherwise ineligible for ASCT, who were randomized to MPT-T or MPRR. A total of 306 participants were randomized with the median age of approximately 76 years old (75.8 in MPT-T; 76.6 in mPR-R) with most having an ECOG of $0-1$ (81.2\% in MPT-T; $80.9 \%$ in mPR-R). At a median followup of 40.7 months, PFS on MPT-T was 21 months and mPR-R was 18.7 months (HR 0.84, 95\% CI 0.64-1.09; $\mathrm{P}=$ $0.186)$. Incorporating age, in those younger than 75 , the PFS for MPT-T was 25.6 months versus 21.6 months for mPR-R. For those 75 or older, PFS for MPT-T was 18.9 months versus 16.6 months for mPR-R. The OS for MPT$\mathrm{T}$ was 52.6 months and mPR-R was 47.7 months $(\mathrm{P}=$ $0.476)$. More grade 3 toxicities were seen with MPT-T (59.5\%) compared to mPR-R (40\%). Quality-of-life measures favored mPR-R over MPT-T. Overall, mPR-R was found to have statistically similar response rates, PFS, and OS to MPT-T with lower rates of toxicity and better quality-of-life, establishing mPR-R as a treatment option for older patients with new diagnoses of multiple myeloma. $^{21}$

\section{MPT-T versus MPR-R (2016)}

Similar to E1A06, this trial performed by Zweegman et al evaluated whether using lenalidomide in place of thalidomide in the MPT regimen improved outcomes for older adults with newly diagnosed multiple myeloma who are ineligible for ASCT. This was an open-label, phase 3 RCT with two arms: MPT-T and MPR-R. A total of 668 participants were randomized to one of the two arms, with each having similar median ages (72 for MPT-T and 73 for MPR-R) and most with WHO performance status of 0-2 (95\% MPT-T; 85\% for MPR-R). Both arms achieved similar PFS: 20 months with MPT-T and 23 months with MPR-R (HR 0.87, 95\% CI 0.72-1.04; $\mathrm{P}=0.12$ ) at a median follow-up of 36 months. There was no significant difference in OS between the two arms at two, three, and four years; OS for MPT-T was 73\%, 64\%, and 52\% versus OS for MPR-R was $84 \%, 69 \%$, and $56 \%$, respectively (HR $0.82,95 \%$ CI $0.64-1.06 ; \mathrm{P}=0.13$ ). Each arm experienced significant grade $3-4$ toxicity ( $81 \%$ in MPT-T vs $86 \%$ in MPR-R; $\mathrm{P}=0.13)$. Expectedly, MPT-T had more neuropathy than MPR-R (16\% vs $2 \% ; \mathrm{P}<0.001)$; this led to participants discontinuing treatment early, resulting in a significantly shorter duration of maintenance in the MPT-T arm (5 months) compared to MPR-R (17 months). Toxicity in MPR-R was notable for more neutropenia
(64\%) than MPT-T $(27 \%)(\mathrm{P}<0.001)$. Both arms had similar rates of infections (19\% in MPR-R vs $21 \%$ in MPT-T; $\mathrm{P}=0.69)$ and VTE (8\% in both arms). This study demonstrated that MPR-R is similar to MPT-T regarding PFS and OS, but MPT-T was associated with clinically significant peripheral neuropathy that inhibited the completion of maintenance therapy. ${ }^{22}$ This study and E1A06 helped establish lenalidomide as having similar efficacy but less toxicity than thalidomide.

\section{Lenalidomide and Dexamethasone-Based Regimens Southwest Oncology Group (SWOG) S0232 (2010)}

SWOG study, S0232, was a phase 3 RCT that examined the use of lenalidomide and dexamethasone compared to placebo and dexamethasone in newly diagnosed multiple myeloma patients who were ineligible for ASCT. In total 198 participants enrolled, with 85\% having an ECOG of $0-1$. Approximately $50 \%$ of total participants were older than 65 years old, with $48(49 \%)$ in the lenalidomide and dexamethasone arm and $45(47 \%)$ in the placebo and dexamethasone arm. Induction included three cycles of lenalidomide $25 \mathrm{mg}$ daily for 28 days or placebo with dexamethasone $40 \mathrm{mg}$ daily on days $1-4,9-12$, and 17-20. The maintenance phase included lenalidomide $25 \mathrm{mg}$ daily for 21 days or placebo and dexamethasone $40 \mathrm{mg}$ daily on days 1-4 and 15-18. Cross-over to the lenalidomide and dexamethasone arm was allowed due to disease progression. ${ }^{23}$ Ultimately, the data and safety monitoring committee closed the study early due to inferior efficacy in the placebo and dexamethasone arm and the preliminary results from E4A03 ECOG, which showed similar efficacy with less toxicity of low-dose compared to high-dose dexamethasone. ${ }^{23,24}$ Final analysis of S0232 revealed lenalidomide and dexamethasone were superior to placebo and dexamethasone for progression-free survival (PFS) (78\% vs 52\%; $\mathrm{P}=0.002)$, overall response rate (ORR) $(78 \%$ vs 48\%; $\mathrm{P}<0.001)$, and VGPR (63\% vs 16\%; P <0.001), while the two arms had similar OS at one-year $(94 \%$ vs $88 \% ; \mathrm{P}=0.25)$. Grade 3 or 4 adverse events were more frequent in the lenalidomide and dexamethasone arm, in particular for neutropenia $(21 \%$ vs $5 \%$; $\mathrm{P}<0.001)$ and venous thromboembolism (VTE) (23.5\% vs 5\%; P <0.001). Three-year follow-up showed continued improved PFS in the lenalidomide and dexamethasone arm compared to placebo 
and dexamethasone arm ( $52 \%$ vs $32 \%)$ but no difference in OS. ${ }^{23}$ It is unclear if older adults fared differently than those under 65 years old, as the results were not stratified by age.

\section{E4A03 Eastern Cooperative Oncology Group (ECOG) Trial: High- versus Low-Dose Dexamethasone (2010)}

Due to concerns over high-dose dexamethasone and its associated toxicity, the E4A03 study evaluated the role of high- versus low-dose dexamethasone in combination with lenalidomide to determine if similar efficacy could be achieved with less toxicity. This was an open-label, noninferiority trial in which patients were randomly assigned to high-dose dexamethasone and lenalidomide versus lowdose dexamethasone and lenalidomide. Lenalidomide dosing was $25 \mathrm{mg}$ daily on days 1-21 out of a 28-day cycle in both arms, with the high-dose arm using dexamethasone $40 \mathrm{mg}$ daily on days $1-4,9-12$, and $17-20$, and the lowdose arm using dexamethasone $40 \mathrm{mg}$ on days $1,8,15$, and 22. The response rate after four cycles was the primary endpoint. A total of 445 patients were randomized to high-dose dexamethasone versus low-dose dexamethasone, with similar patient characteristics in median age (66 versus 65 , respectively) and ECOG $(0-1$ in $91 \%$ of participants in each arm). Patients were able to later undergo ASCT or continue therapy at the discretion of the investigator. The overall response rate (complete response + partial response) after four cycles was better in the high-dose dexamethasone compared to the low-dose arm (79\% vs $68 \%$, respectively). However, over the first four cycles, the high-dose arm had significantly greater toxicity, including VTE (26\% vs $12 \% ; \mathrm{P}=0.0003)$ and pneumonia $(16 \%$ vs $9 \% ; \mathrm{P}=0.04)$, and more deaths ( $\mathrm{n}=12$ vs $\mathrm{n}=1 ; \mathrm{P}=0.003$ ). At one year, the OS was better for the low-dose dexamethasone arm (96\%) compared to the high-dose arm (87\%) $(\mathrm{P}=0.0002)$. The study was stopped, and high-dose dexamethasone participants crossed-over to the low-dose arm. Of note, while not an initial study endpoint, there were differences in OS depending on age. One-year OS for high-dose versus lowdose dexamethasone in those under age 65 was $91 \%$ versus $98 \%$, respectively; in those over age 65 , the oneyear OS on high-dose versus low-dose was $83 \%$ versus $94 \%{ }^{24}$ The E4A03 trial was pivotal for showing while high-dose dexamethasone achieved improved response rates, it did not translate into improved OS and was particularly toxic in older adults.

\section{FIRST Trial (20I4)}

Although lenalidomide was gaining traction in the treatment of older adults with multiple myeloma at this time, the regimen of melphalan, prednisone, and thalidomide (MPT) was still the standard of care for transplantineligible patients until the FIRST trial. The FIRST trial evaluated lenalidomide and dexamethasone (Rd) versus MPT in patients 65 and older or were otherwise ineligible for ASCT. This was a phase 3, open-label RCT with three arms: Rd continuing until progression, $\mathrm{Rd}$ for 72 weeks (18 cycles), and MPT for 72 weeks (12 cycles). For the Rd arms, lenalidomide was administered $25 \mathrm{mg}$ daily on days 1-21 and dexamethasone $40 \mathrm{mg}$ ( $\leq 75$ years old) or $20 \mathrm{mg}$ ( $>75$ years old) was administered on days $1,8,15$, and 22 out of a 28-day cycle. For the MPT arm, melphalan $0.25 \mathrm{mg} / \mathrm{kg}$ ( $\leq 75$ years old) or $0.20 \mathrm{mg} / \mathrm{kg}$ ( $>75$ years old) was given on days $1-4$, prednisone $2 \mathrm{mg} / \mathrm{kg}$ on days $1-4$, and thalidomide $200 \mathrm{mg}$ daily ( $\leq 75$ years old) or $100 \mathrm{mg}$ daily ( $>75$ years old) was given in 42-day cycles. A total of 1623 patients were randomized, with 535 in the continuous Rd arm, 541 in the 18-cycle Rd arm, and 547 in the MPT arm. The median age in the 3 arms was 73 years old, with approximately $35 \%$ in each arm over 75 years old; nearly all participants had an ECOG of $0-2$. The PFS of continuous Rd (25.5 months) was significantly better than 18-cycles of $\mathrm{Rd}$ (20.7 months; HR 0.7 for risk of progression or death; $\mathrm{P}<0.001)$ and MPT $(21.2$ months; HR 0.72; $\mathrm{P}<0.001)$. The interim OS analysis for each arm found the OS for continuous $\mathrm{Rd}$ was $70 \%$ (3 years) and 59\% (4 years); OS for 18-cycles Rd was $66 \%$ (3 years) and 56\% (4 years); OS for MPT was $62 \%$ (3 years) and $51 \%$ (4 years). There was no significant difference in the OS for each arm $(\mathrm{P}<0.0096$; did not meet prespecified barrier for significance); however, continuous $\mathrm{Rd}$ reduced the risk of death when compared to MPT (HR $0.78 ; \mathrm{P}=0.02$ ). Each arm had similar rates of grade $3-4$ toxicity: $85 \%$ in continuous $\mathrm{Rd}, 80 \%$ in 18 -cycle $\mathrm{Rd}$, and $89 \%$ in MPT. The MPT arm had higher rates of neutropenia (45\%) compared to the Rd arms ( $28 \%$ for continuous, $26 \%$ for 18 -cycles) but the $\mathrm{Rd}$ arms had more frequent infections ( $29 \%$ for continuous, $22 \%$ for 18 -cycles) than MPT (17\%). Second primary cancers were noted in each arm, with $3 \%$ in continuous $\mathrm{Rd}, 5 \%$ in 18 -cycles Rd, and $6 \%$ in MPT, with hematologic malignancies being more common in $\mathrm{MPT}^{25}$ The final analysis of the FIRST trial at 
a median follow-up of 5.6 years revealed that OS was significantly longer with continuous Rd (59.1 months) compared to MPT (49.1 months) (HR 0.78; 95\% CI 0.67-0.92; $\mathrm{P}=0.0023$ ), and OS was similar between continuous $\mathrm{Rd}$ and 18-cycles of $\mathrm{Rd}$ (62.3 months) (HR 1.02; 95\% CI $0.86-1.2) .{ }^{26}$ The FIRST trial set the standard of $\mathrm{Rd}$ as a first-line treatment option for older adults.

\section{Triplet vs Doublet Lenalidomide-Regimens (2016)}

Expanding upon the work in the FIRST trial, Margarotto et al analyzed the PFS of lenalidomide containing threedrug regimens (triplet) versus two-drug regimens (doublet) for older adults with new diagnoses of multiple myeloma who were ineligible for ASCT. This was a phase 3 RCT with three arms: cyclophosphamide, prednisone, and lenalidomide (CPR); melphalan, prednisone, and lenalidomide (MPR); lenalidomide and dexamethasone (Rd). The induction phase included 28day cycles for 9 cycles with MPR using melphalan $0.18 \mathrm{mg} / \mathrm{kg}$ daily for 4 days or $0.13 \mathrm{mg} / \mathrm{kg}$ daily (in those older than 75) on days $1-4$, prednisone $1.5 \mathrm{mg} / \mathrm{kg}$ daily on days $1-4$, and lenalidomide $10 \mathrm{mg}$ daily on days 1-21. Initially, CPR patients received the same dose of lenalidomide, cyclophosphamide $50 \mathrm{mg}$ PO every other day for 28 days (or 21 days if over age 75), and prednisone $25 \mathrm{mg}$ every other day; this was later adjusted for those between ages $65-75$ to lenalidomide $25 \mathrm{mg}$ daily and cyclophosphamide $50 \mathrm{mg}$ daily for 21 days. The $\mathrm{Rd}$ arm was treated with lenalidomide $25 \mathrm{mg}$ daily on days 1-21 and dexamethasone $40 \mathrm{mg}$ on days 1, 8, 15, and 22 (or $20 \mathrm{mg}$ in those older than age 75). The maintenance phase included lenalidomide $10 \mathrm{mg}$ on days 1-21 or with prednisone $25 \mathrm{mg}$ every other day continuously. A total of 654 participants were randomized with a median age of 73 in the CPR and $\mathrm{Rd}$ arms and 74 in the MPR arm; approximately $25 \%$ in each arm had their fitness described as frail. There was no significant difference in PFS between triplet (22 months) and doublet (21 months) regimens $(\mathrm{P}=0.284)$ at a median follow-up of 39 months. The four-year OS was also not significantly different, with triplet OS of $67 \%$ and doublet OS of $58 \%(\mathrm{P}=0.709)$. The only significant difference in toxicity was a higher rate of neutropenia in the MPR arm (64\%) compared to CPR (29\%) and $\mathrm{Rd}(25 \%)(\mathrm{P}<0.001)$. A post hoc analysis found a PFS advantage with MPR in fit patients versus $\mathrm{Rd}$
(HR 0.671, $\mathrm{P}=0.037$ ) and $\mathrm{CPR}$, which was not appreciated in intermediate or frail patients. Overall, outcomes with $\mathrm{Rd}$ were similar to the triplet regimens in older patients with multiple myeloma, expanding upon the findings of the FIRST trial. ${ }^{27}$

\section{$R d-R$ versus $R d$ in Intermediate-Fit Patients (2018)}

In order to better capture a "real world" patient population, a phase 3 RCT, RV-MM-PI-0752, evaluated the impact of dose-adjusted multiple myeloma directed therapy in newly diagnosed older adults who were intermediate-fit by the International Myeloma Working Group (IMWG) Frailty score. This data was presented at the American Society of Hematology in 2018 and is awaiting final publication. Patients were randomized to one of two arms: Rd-R or continuous $\mathrm{Rd}$. The Rd-R arm included a total of nine 28day cycles of lenalidomide $25 \mathrm{mg} /$ day for 21 days and dexamethasone $20 \mathrm{mg}$ on days $1,8,15,22$, followed by lenalidomide maintenance $10 \mathrm{mg} /$ day for 21 days. The continuous $\mathrm{Rd}$ arm included lenalidomide $25 \mathrm{mg}$ /day for 21 days and dexamethasone $20 \mathrm{mg}$ on days 1, 8, 15, 22 . Treatment continued in both arms until disease progression, and the primary endpoint was EFS. Nearly 200 patients were enrolled, with 98 in the Rd-R arm and 101 in the continuous Rd arm. Patient characteristics were well balanced between arms, with a median age of 75 (range 73-77) in Rd-R and 76 (range 74-79) in continuous $\mathrm{Rd}$ ( $\mathrm{P}=0.06)$, and a similar distribution of intermediate-fit for age ( $\geq 76$ ) patients, with $47 \%$ in Rd-R vs $57 \%$ in continuous Rd. An impairment in Charlson Index, ADL or IADL was seen in $53 \%$ versus $43 \%$ of patients, respectively. At a median follow-up of 25 months, EFS was 9.3 versus 6.6 months in Rd-R versus continuous Rd (HR 0.72, 95\% CI $0.52-0.99, \mathrm{P}=0.04)$. There were no significant differences in PFS (18.3 versus 15.5 months) or OS (85\% versus $81 \%$ ) at 18 months. Adverse events, including neutropenia, infections, and dermatologic reactions, were less frequent in $\mathrm{Rd}-\mathrm{R}$ than continuous $\mathrm{Rd}$ (30 versus 39\%), although the difference was not significant. Dose reductions in lenalidomide after 9 cycles $(1 \%$ versus $21 \%, \mathrm{P}=0.06)$ and dexamethasone $(17 \%$ versus $29 \%, \mathrm{P}=0.06)$ were needed less often in Rd-R than continuous $\mathrm{Rd}^{28}$ This trial shows that an adjusted schedule of Rd-R is feasible in intermediatefit, older adults with multiple myeloma, and is one of the first trials to incorporate the IMWG Frailty score in a prospective manner. 


\section{Lenalidomide and Dexamethasone in Relapsed/Refractory Disease}

While we recognize that these studies may not be as relevant to those who receive lenalidomide in upfront therapy, older patients with relapsed/refractory disease may still be exposed to lenalidomide. This may be particularly true in Europe and the United Kingdom, where practice patterns may differ in part due to medication cost and the later timing of approval for the use of lenalidomide in the first-line setting ( 2015 by the European Medicines Agency; 2019 by the National Institute for Health and Care Excellence). ${ }^{29-31}$

\section{RevLite (2017)}

Due to concern about treatment-related toxicities, the RevLite study evaluated the impact of starting lowerdose lenalidomide and lower-dose dexamethasone in older patients with multiple myeloma in the relapsed/ refractory setting. This was a single-arm, Phase 2 trial to assess the tolerability and efficacy of lower-dose lenalidomide and dexamethasone ( $\mathrm{rd}$ ). The treatment included lenalidomide $15 \mathrm{mg}$ daily on days 1-21 and dexamethasone $20 \mathrm{mg}$ on days $1-4,9-12$, and $17-20$ of a 28 -day cycle over the first four cycles, and then dexamethasone $20 \mathrm{mg}$ on days $1-4$ only starting with cycle 5 . Treatment continued until disease progression or intolerable adverse effects. The median age of the 149 participants was 69 (70.5\% were 65 years old or older). A matched cohort of participants from the MM-009 and MM-010 studies who were treated with lenalidomide $25 \mathrm{mg}$ and dexamethasone $40 \mathrm{mg}$ (RD) were used as comparison. They found there was no difference in PFS or OS with the matched cohort: PFS was 8.9 months in rd versus 11 months in $\mathrm{RD}(\mathrm{P}=$ 0.336), and the OS for rd was 30.5 months compared to 34.8 months for $\mathrm{RD}(\mathrm{P}=0.21)$. Grade 3-4 toxicities were less common in rd compared to $\mathrm{RD}$, including rates of infection ( $23 \%$ vs $31 \%$ ), VTE ( $3 \%$ vs $13 \%$ ) and neutropenia $(29 \%$ vs $41 \%) .{ }^{32}$ While the lack of an RCT design makes it challenging to draw definitive conclusions, this study suggests that there may be a role for reduced dose lenalidomide and dexamethasone in older patients with medical comorbidities.

\section{Real-World Lenalidomide Dosing (2017)}

While reductions in lenalidomide dosing have been described in clinical trials, little was known about the realworld experience of lenalidomide dosing in older adults with relapsed multiple myeloma until a study by Nakaya et al. This was a retrospective cohort study that analyzed patients age 65 or older who were treated with lenalidomide in the relapsed setting between January 2011 and December 2015. During the study period, 56 patients were treated with lenalidomide on days 1-21 and dexamethasone (20 mg or $40 \mathrm{mg}$ ) on days $1,8,15$ and 22. They found most patients were started and continued a lenalidomide dose below the recommended dose of $25 \mathrm{mg}$ daily. The most common starting doses per day were lenalidomide $10 \mathrm{mg}(45 \%), 5 \mathrm{mg}(21 \%), 15 \mathrm{mg}$ (20\%), $25 \mathrm{mg}(10 \%)$, and $20 \mathrm{mg}(4 \%)$. The most frequent continued treatment doses per day were $10 \mathrm{mg}(46 \%)$, $5 \mathrm{mg}(25 \%), 15 \mathrm{mg}$ (16\%), $25 \mathrm{mg} \mathrm{(9 \% ),} \mathrm{and} 20 \mathrm{mg}$ (4\%). The overall median time to progression was 11.8 months, and there was no significant difference between those treated with lower or higher dose lenalidomide; median time to progression was 14.5 months with lenalidomide 5-10 mg daily, and the median was 8.9 months for those treated with $>10 \mathrm{mg}$ daily $(\mathrm{P}=0.466)$. Median $\mathrm{OS}$ for the cohort was 39.2 months, and again there was no significant difference between the lower and higher dose lenalidomide; median OS on lenalidomide 5-10 mg daily was 38.9 months, and median OS on $>10 \mathrm{mg}$ lenalidomide was not reached $(\mathrm{P}=0.275)$. Response rates were reasonable, with the ORR of $73 \%$ with $36 \%$ achieving a VGPR or better. The primary reasons for dose limitations included renal dysfunction (54\%), $\geq$ grade 3 fatigue (20\%), zgrade 3 hematologic disorder (14\%), and $\geq$ grade 3 rash (14\%). As high as $16 \%$ of patients developed a second malignancy during treatment. ${ }^{33}$ This singleinstitution, retrospective study indicates that many patients have challenges tolerating the recommended doses of lenalidomide outside of a clinical trial setting, and outcomes for lower dose lenalidomide were similar to higher dose lenalidomide in this limited cohort.

\section{Lenalidomide and Bortezomib-Based Regimens SWOG S0777 (2017)}

SWOG S0777 evaluated whether the standard treatment for newly diagnosed multiple myeloma patients not immediately undergoing ASCT (Rd) could be improved by adding bortezomib. This was an open-label, phase $3 \mathrm{RCT}$ with two arms: bortezomib plus Rd (VRd) and Rd alone. VRd induction included bortezomib $1.3 \mathrm{mg} / \mathrm{m}^{2}$ on days 1 , 4,8 and 11 , lenalidomide $25 \mathrm{mg}$ on days $1-14$, and 
dexamethasone $20 \mathrm{mg}$ on days $1-2,4-5,8-9,11-12$, given in 21-day cycles for a total of 8 cycles. $\mathrm{Rd}$ induction included lenalidomide $25 \mathrm{mg}$ daily on days $1-21$ and dexamethasone $40 \mathrm{mg}$ on days $1,8,15$, and 22 in 28-day cycles for a total of 6 cycles. Both arms received the same maintenance regimen of lenalidomide $25 \mathrm{mg}$ daily on days 1-21 and dexamethasone $40 \mathrm{mg}$ on days $1,8,15$, and 22 . A total of 525 patients were randomized, with a median age of 63 in each arm; $43 \%$ of all participants were age 65 or older, with $38 \%$ in the VRd arm and $48 \%$ in the Rd arm. The primary endpoint of PFS was significantly improved in the VRd arm (43 months) compared to the Rd arm (30 months) (HR 0.712, 96\% CI 0.56-0.906; $\mathrm{P}=0.0018$ ). Median OS was also significantly better for the VRd arm (75 months) than the Rd arm (64 months) (HR 0.709, 95\% CI 0.524-0.959; $\mathrm{P}=0.025)$. Each arm experienced a similar number of grade 3-4 toxicities, including similar rates of cytopenias, infections, and vascular problems. The primary grade 3 toxicity was peripheral neuropathy, which was significantly worse with VRd than Rd (33\% vs $11 \%$; $\mathrm{P}<0.0001){ }^{34} \mathrm{~S} 0777$ supported adding bortezomib to upfront treatment in those patients not immediately undergoing ASCT, although it should be noted that it was not performed exclusively in an older patient population.

\section{RVD-Lite (2018)}

Building off of the SWOG S0777 and RevLite studies, RVD-lite was a single-arm, phase 2 study designed to evaluate the efficacy of reduced dose RVD in older patients with multiple myeloma who were ineligible for ASCT. Induction included lenalidomide $15 \mathrm{mg}$ daily on days $1-21$, bortezomib $1.3 \mathrm{mg} / \mathrm{m}^{2}$ on days $1,8,15$, and 22 , and dexamethasone $20 \mathrm{mg}$ on days $1-2,8-9,15-16$, 22-23 (in patients 65-76 years old) and on days 1, 8, 15, 22 (for patients older than 75) in 35-day cycles for a total of 9-cycles. Consolidation included lenalidomide $15 \mathrm{mg}$ daily on days $1-21$ and bortezomib $1.3 \mathrm{mg} / \mathrm{m}^{2}$ on days 1 and 15 in 28-day cycles for a total of 6 cycles. A total of 50 participants were enrolled with a median age of 73 at the time of diagnosis, and most had an ECOG 0-1 (86\%). The ORR after 4 cycles was $86 \%$, with $66 \%$ of participants achieving a VGPR or better. Median follow-up was 30 months, at which time the median PFS was 35.1 months (95\% CI: 30.9 - not reached) and median OS was not reached. As high as $78 \%$ of patients required dose adjustments, including dexamethasone (64\%), lenalidomide (54\%), and bortezomib (38\%). Peripheral neuropathy of any grade was seen in $62 \%$ of patients and was mostly grade $1-2(n=25)$ with 1 patient developing grade 3 peripheral neuropathy. The most frequent grade 3-4 toxicities included hypophosphatemia (34\%), fatigue (16\%), neutropenia (14\%), and rash (10\%). Quality-oflife metrics showed improvements in physical functioning $(\mathrm{P}=0.013)$ and disease symptoms $(\mathrm{P}=0.001)$ but no significant difference in global functioning at the end of treatment compared to baseline. The results of RVD-lite suggest that reduced-dose RVD is effective with low levels of toxicity, leading to stable or improved quality-of-life. ${ }^{35}$ Given the lack of an RCT design, the interpretation of the results should be interpreted with caution, but RVD-lite provides support to using reduced-dosing RVD in select older patients.

\section{Lenalidomide and Daratumumab- Based Regimens MAIA Trial (2019)}

The MAIA trial was the first to incorporate a monoclonal antibody, daratumumab, into first-line treatment for older patients with multiple myeloma who are ineligible for ASCT. The objective of the MAIA trial was to evaluate the safety and efficacy of adding daratumumab to Rd (Dara$\mathrm{Rd}$ ) in comparison to $\mathrm{Rd}$ alone. This was an open-label, phase 3 RCT with two arms, with Rd serving as the control for the Dara-Rd arm. Each arm was treated with lenalidomide $25 \mathrm{mg}$ daily on days 1-21 and dexamethasone $40 \mathrm{mg}$ (or $20 \mathrm{mg}$ if over age 75) on days 1, 8, 15, and 22 until disease progression or intolerable adverse effects. The Dara-Rd arm was also treated with daratumumab $16 \mathrm{mg}$ / $\mathrm{kg}$ once per week during cycles $1-2$, every 2 weeks during cycles 3-6, and then every 4 weeks indefinitely. A total of 737 patients were randomized to one of the two arms, with each arm having a similar median age (73 for the daratumumab arm, 74 for Rd alone) with almost half of all patients 75 years or older $(43.5 \%$ in the daratumumab arm, $43.6 \%$ in the Rd alone arm). Most patients had an ECOG performance status of $0-1$, with $17.1 \%$ having an ECOG of 2 or higher in the Dara-Rd and $16 \%$ in the Rd alone arm. At median follow-up of 28 months, PFS in the Dara-Rd (endpoint not reached) was longer than the Rd alone arm (31.9 months) (for disease progression or death, HR $0.56,95 \% \mathrm{CI}$ $0.43-0.73 ; \mathrm{P}<0.001)$. Median OS was not reached in either arm, although at the time of analysis, $16.8 \%$ of patients in the Dara-Rd arm died versus $20.6 \%$ in the Rd alone group. Compared to the $\mathrm{Rd}$ alone arm, more patients in the DaraRd arm achieved a CR (47.6\% vs $24.9 \%$; $<0.001)$, VGPR 
(53.1\% vs $79.3 \% ; \mathrm{P}<0.001)$ and achieved negative minimal residual disease ( $7.3 \%$ vs $24.2 \%$; $<<0.001)$. Both arms had similar serious adverse events $(62.9 \%$ with Dara-Rd vs $62.7 \%$ with $\mathrm{Rd}$ ), as well as similar death rates from adverse events (6.9\% with Dara-Rd vs $6.3 \%$ with Rd). Grade 3 or 4 toxicities were notable for neutropenia (50\% with Dara-Rd vs $35.3 \%$ with $\mathrm{Rd}$ ), anemia ( $11.8 \%$ vs $19.7 \%$ ), and infections $(32.1 \%$ vs $23.3 \%)$, including pneumonia ( $13.7 \%$ vs $7.9 \%)$. Overall the combination of Dara-Rd was found to have improved PFS and response rate but was also notable for a higher incidence of infections and neutropenia. ${ }^{36}$ The superiority of the daratumumab-lenalidomide and dexamethasone combination led to the approval of this regimen in the United States in 2019.

\section{CEPHEUS: Ongoing Trial Using Daratumumab in Older Adults}

An ongoing trial, CEPHEUS (NCT03652064) is also evaluating the role of daratumumab in the first-line setting in older patients with newly diagnosed multiple myeloma. CEPHEUS is a phase $3 \mathrm{RCT}$ evaluating D-VRd versus VRd in patients who are not being considered for transplant as initial therapy, with patient stratification including those age $<70$ and transplant-ineligible, age $<70$ and refused transplant, or age $\geq 70$. All patients will be treated with eight 21-day cycles of VRd, followed by 28-day cycles of Rd. Patients randomized to the D-VRd arm will also be given weekly daratumumab in Cycles 1-2, every 3 weeks in Cycles 3-8, and every 4 weeks starting in cycle 9 until disease progression. The primary endpoint is the rate of minimal residual disease by next-generation sequencing. ${ }^{37}$ The CEPHEUS trial is anticipated to provide valuable information about the safety and efficacy of D-VRd in older adults who are newly diagnosed with multiple myeloma.

\section{Toxicities}

The use of lenalidomide in multiple myeloma has led to improvements in PFS and OS, but it is also associated with known toxicities as described in clinical trials. The following lenalidomide-associated toxicities are the most notable in older patients with multiple myeloma.

\section{Venous Thromboembolism (VTE)}

The development of VTE in multiple myeloma patients on lenalidomide was first noted in early trials. In MM-010, which evaluated the use of lenalidomide and dexamethasone versus dexamethasone alone in relapsed/refractory multiple myeloma, lenalidomide was associated with higher rates of deep venous thrombosis ( $4 \%$ vs $3.5 \%$ ) and pulmonary embolism $(4.5 \%$ vs $1.2 \%)$ than placebo. ${ }^{38}$ Interim multivariate analysis of MM-010 and another identical trial showed an increase in VTE with lenalidomide plus high-dose dexamethasone (OR 3.51, 95\% CI 1.77-6.97), which was also appreciated with concurrent erythropoietin administration (OR 3.21, 95\% CI 1.72-6.01). In those same trials, patients who were concurrently treated with salicylates did not develop VTE. ${ }^{39}$ The E4A03 trial also found a higher incidence of VTE in patients treated with lenalidomide plus high-dose dexamethasone compared to lenalidomide plus low-dose dexamethasone. ${ }^{24,40}$ The National Comprehensive Cancer Network (NCCN) guidelines recommend using VTE prophylaxis with aspirin $81 \mathrm{mg}$ or $325 \mathrm{mg}$ for all patients on IMiDs, with anticoagulation recommended for high-risk patients. ${ }^{41}$ Given the known association between lenalidomide and VTE, risk assessment models have recently been developed and validated to predict the risk of VTE in patients with multiple myeloma. One such model is the IMPEDE VTE score, which predicts the risk of VTE development in patients with new diagnoses of multiple myeloma based on criteria listed in Table 1. The criteria were derived from a retrospective analysis of patients from the Veterans Administration Central Cancer Registry and externally validated using patients in the Surveillance, Epidemiology, End Results-Medicare database. As the IMPEDE VTE score increased, there was a corresponding increase in the rate of VTE (HR 1.20 per point, $\mathrm{P}<0.0001$ ). Those with an IMPEDE VTE score $\geq 8$ had a cumulative VTE rate of $15 \%$ at 6 months. ${ }^{42}$ A retrospective cohort analysis also recently externally validated the IMPEDE VTE score and used the total score to stratify patients into three distinct groups: lowrisk (score $<3$ ), intermediate-risk (score 3-6), and high-risk (score $>6$ ). ${ }^{43}$ Such a risk score may help identify older patients with multiple myeloma at risk for VTE development before starting treatment.

\section{Cytopenias}

One of the most common grade 3 or higher toxicities in phase 3 clinical trials were cytopenias, particularly neutropenia and thrombocytopenia. ${ }^{17,20,22,23,34,36}$ This is most likely to occur within the first several months of treatment with lenalidomide and guidelines provide recommendations on how to best dose-adjust based on the degree of cytopenia. ${ }^{44,45}$ 
Table I IMPEDE VTE: A Risk Model for VTE Development in Patients with Multiple Myeloma

\begin{tabular}{|l|l|}
\hline Variable & Score \\
\hline Immunomodulatory drug & +4 \\
Body Mass Index $\geq 25 \mathrm{~kg} / \mathrm{m}^{2}$ & +1 \\
Pelvic, hip, or femur fracture & +4 \\
Erythropoiesis-stimulating agent & +1 \\
Doxorubicin & +3 \\
Dexamethasone Dose & \\
$\quad$ High-Dose & +4 \\
Low-Dose & +2 \\
Ethnicity/Race = Asian/Pacific Islander & -3 \\
History of VTE before MM & +5 \\
Tunneled line/central venous catheter & +2 \\
Existing thromboprophylaxis: therapeutic LWMH or & -4 \\
warfarin & \\
Existing thromboprophylaxis: prophylactic LWMH or & -3 \\
warfarin & \\
\hline
\end{tabular}

Abbreviations: VTE, venous thromboembolism; MM, multiple myeloma; LWMH, low weight molecular heparin.

\section{Second Primary Malignancies}

Several studies reported an increased risk of developing a second primary malignancy after treatment with lenalidomide in a melphalan-containing regimen or as maintenance post-ASCT. ${ }^{20,25,33,46}$ A pooled analysis of 11 clinical trials of relapsed multiple myeloma treated with lenalidomide regimens showed an incidence rate of 3.62 with hematologic/ solid tumors having an incidence rate of 2.08 (comparable to background incidence). An analysis of MM-009 and MM010 showed an incidence rate of 3.98; excluding nonmelanoma skin cancers, the incidence rate for hematologic/ solid cancers was 1.71 on Rd. However, this was compared to 0.91 on placebo/dexamethasone. ${ }^{47}$ As a consequence, the NCCN guidelines recommend that all patients be counseled on the potential risk of developing second primary malignancies prior to starting lenalidomide. ${ }^{41}$

\section{Comprehensive Geriatric Assessment and Frailty}

While most trials use age as a guide when determining treatment eligibility, doing so may exclude sections of fit, older adults who may benefit from new treatments. A comprehensive geriatric assessment provides the opportunity to evaluate several aspects of older patients' health as chronological age is not necessarily reflective of physiologic age. A comprehensive geriatric assessment includes evaluation of functional status, cognition, fall history, sensation, nutrition, social support, psychosocial status, and medical comorbidities, and can help provide a comprehensive evaluation of the patient. ${ }^{5,48}$ One approach to operationalizing the heterogeneous health status of older adults with myeloma is the IMWG Frailty score, which incorporates a patient's functional status and comorbidities. The frailty score was developed through a pooled analysis of baseline assessments in 869 newly diagnosed older multiple myeloma patients who participated in phase 3 clinical trials. The median age was $74 \%$ and $46 \%$ were older than 75 . The baseline assessment included the Katz Activity of Daily Living, the Lawton Instrumental Activity of Daily Living, and the Charlson Comorbidity Index. A scoring system was then created using age, medical comorbidities, and functional status. This resulted in three categories of patients: fit (39\%), intermediate $(31 \%)$ and frail $(30 \%)$, which were significantly associated with OS; at three years, the OS in patients categorized as fit was $84 \%, 76 \%$ in patients categorized as intermediate-fit, and $57 \%$ in those categorized as frail. ${ }^{49}$

While the IMWG frailty approach has gained much traction, other approaches to characterizing vulnerability in older adults with myeloma have been developed and applied in older adults with multiple myeloma as predictors of toxicity, progression-free and overall survival. These include the Revised Myeloma Comorbidity Index (which incorporates performance status, specific comorbidities, and the frailty phenotype), ${ }^{50}$ the simplified frailty scale (which comprises age, performances status, and comorbidities), ${ }^{51}$ and the Myeloma Research Alliance Risk Profile (which includes age, performances status, and International Staging System Stage and utilizes the inflammatory biomarker C-reactive protein). ${ }^{52}$ The incorporation of assessment of geriatric domains in older patients with multiple myeloma should be considered, as they have the potential to provide better insight into what patients benefit from particular therapies. ${ }^{53}$

\section{Evolving Treatment Options for Older Adults}

Although lenalidomide, either alone or in combination, is a part of the current treatment standard for older adults, the treatment landscape for myeloma is rapidly evolving. It is likely that the role of lenalidomide will continue to evolve as new drugs and combinations demonstrate efficacy in older adults with myeloma, whether it be through novel drug combinations as in the CEPHEUS trial, ${ }^{37}$ or through lenalidomide-free regimens. The ALCYONE trial showed the efficacy of a lenalidomide-free regimen by using the combination of daratumumab plus bortezomib, melphalan, 


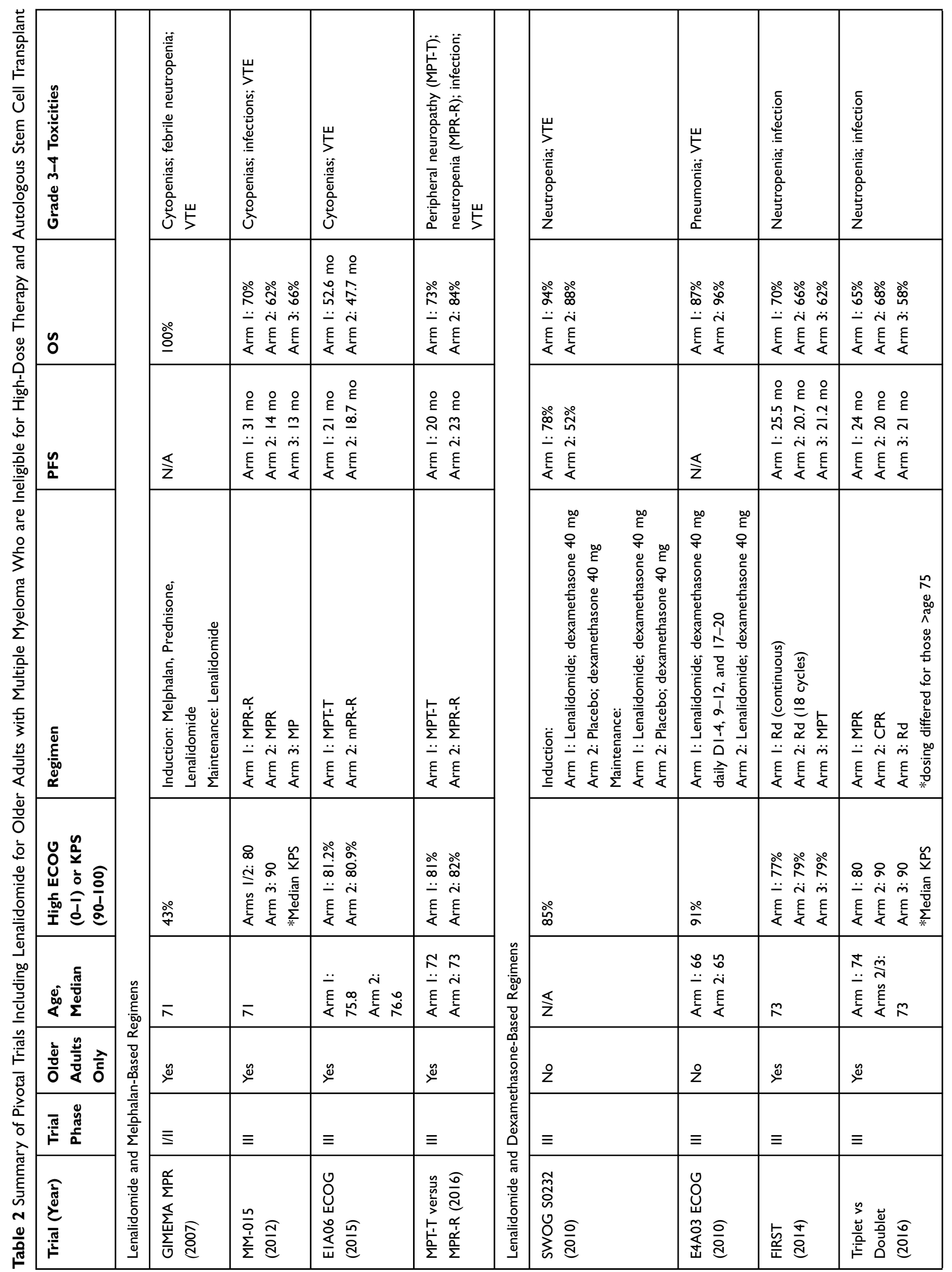




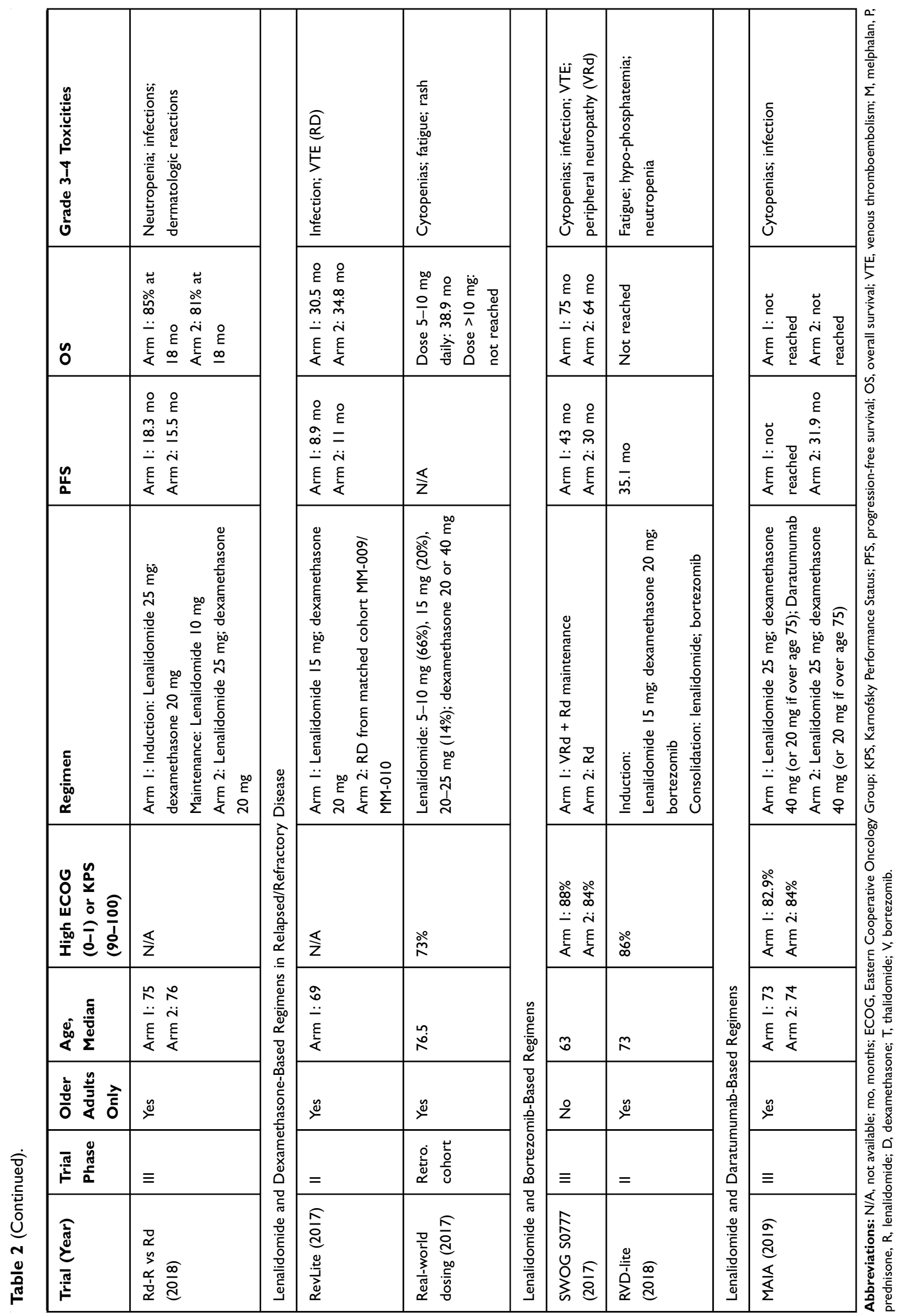


and prednisone (D-VMP) in newly diagnosed, older multiple myeloma patients. The ALCYONE trial was an openlabel, phase 3 RCT that randomized participants to D-VMP or VMP alone. A total of 706 participants were randomized with a median age of 71 in each arm; participants with ECOG 2 were present in both arms $(25.7 \%$ with daratumumab, 23.6\% with VMP). At 18 months, the DVMP had significantly longer PFS (71.6\%) than VMP $(50.2 \%$ ) (HR for disease progression or death $0.5 ; 95 \%$ CI 0.38-0.65; $\mathrm{P}<0.001)$, and the ORR with D-VMP was $90.9 \%$ versus $73.9 \%$ with VMP $(\mathrm{P}<0.001)$. D-VMP had more grade 3-4 infections (23.1\%) than VMP (14.7\%), but both arms had similar rates of grade 3-4 cytopenias. ${ }^{54}$ This regimen was FDA-approved in the United States in May 2018 based on the ALCYONE results. ${ }^{55}$ Long-term follow-up at 40.1 months showed improved OS with D-VMP compared to VMP (HR for death 0.60, 95\% CI $0.46-0.80 ; p=0.0003)$ with a 36-month OS rate of $78 \%$ $(95 \%$ CI $73.2-82) \quad$ versus $67.9 \% \quad(62 \cdot 6-72 \cdot 6)$, respectively. ${ }^{56}$ While the results support a viable lenalidomide-free treatment regimen, a direct comparison of this regimen versus lenalidomide-containing regimens has not been conducted.

The focus of this review was specifically on the use of lenalidomide in older patients with multiple myeloma, and as exemplified by the ALCYONE trial, comprehensive treatment algorithms for older adults may not include entirely lenalidomide-based regimens. Lenalidomide may be challenging to administer to patients with cytopenias given its associated toxicity. ${ }^{44}$ As exemplified in the "real world" studies included here, many older adults are not able to tolerate the full dose of lenalidomide recommended in clinical trials, and dose adjustments are frequently needed. ${ }^{33}$ A recently published comprehensive treatment algorithm for newly diagnosed older adults with multiple myeloma suggested treatment based on breakdown by IMWG Frailty score. Recommended lenalidomide-based regimens included Dara-Rd and VRd for fit patients; Rd$\mathrm{R}, \mathrm{Rd}$, or vrd-lite for intermediate-fit patients; rd for frail patients. Reductions in lenalidomide dosing are recommended for less fit patients, and there are nonlenalidomide-based regimens included in the algorithm as well. ${ }^{57}$ Trials to validate an approach using frailty measures to adjust are underway (NCT03720041). Individual patient characteristics, such as the presence of cognitive impairment, which may impact adherence to oral medication, or renal dysfunction, play a significant role when determining the treatment regimen for older adults with multiple myeloma, which emphasizes the need for an individualized approach.

\section{Conclusion}

The introduction of lenalidomide significantly shifted the treatment paradigm for multiple myeloma, leading to significant improvements in response rates, PFS and OS in the firstline and relapsed/refractory settings. Lenalidomide's significant activity and manageable toxicity profile has made its use particularly important for older patients with multiple myeloma who are ineligible for ASCT. While many of the key clinical trials for multiple myeloma have not been exclusively performed in older adults, several of the trials highlighted in this review focused on the management of older adults with multiple myeloma. While age and performance status were important markers for most of these trials, incorporation of a comprehensive geriatric assessment could provide a more accurate depiction of this patient population and is an area for future exploration.

\section{Author Contributions}

All authors contributed to design of the manuscript, data analysis, drafting and revising the article, gave final approval of the version to be published, and agree to be accountable for all aspects of the work.

\section{Disclosure}

Dr. Wildes receives research funding from Janssen and has served as a consultant for and received personal fees from Seattle Genetics and Carevive Systems. The authors report no other conflicts of interest in this work.

\section{References}

1. Turesson I, Velez R, Kristinsson SY, Landgren O. Patterns of multiple myeloma during the past 5 decades: stable incidence rates for all age groups in the population but rapidly changing age distribution in the clinic. Mayo Clinic Proc. 2010;85(3):225-230. doi:10.4065/ mcp. 2009.0426

2. Costa LJ, Brill IK, Omel J, et al. Recent trends in multiple myeloma incidence and survival by age, race, and ethnicity in the United States. Blood Adv. 2017;1(4):282-287. doi:10.1182/bloodadvances.2016 002493

3. Smith BD, Smith GL, Hurria A, et al. Future of cancer incidence in the United States: burdens upon an aging, changing nation. JCO. 2009;27 (17):2758-2765. doi:10.1200/JCO.2008.20.8983

4. Warren JL, Harlan LC, Stevens J, Little RF, Abel GA. Multiple myeloma treatment transformed: a population-based study of changes in initial management approaches in the United States. JCO. 2013;31 (16):1984-1989. doi:10.1200/JCO.2012.46.3323

5. Rosko A, Giralt S, Mateos M-V, Dispenzieri A. Myeloma in Elderly Patients: when Less Is More and More Is More. Am Soc Clin Oncol Educ Book. 2017;37(37):575-585. doi:10.14694/EDBK_175171 
6. Kumar SK, Dispenzieri A, Lacy MQ, et al. Continued improvement in survival in multiple myeloma: changes in early mortality and outcomes in older patients. Leukemia. 2014;28(5):1122-1128. doi:10.1038/leu.2013.313

7. Child JA, Morgan GJ, Davies FE, et al. High-dose chemotherapy with hematopoietic stem-cell rescue for multiple myeloma. $N$ Engl J Med. 2003;348(19):1875-1883. doi:10.1056/NEJMoa022340

8. Attal M, Harousseau J-L, Stoppa A-M, et al. A prospective, randomized trial of autologous bone marrow transplantation and chemotherapy in multiple myeloma. N Engl J Med. 1996;335(2):91-97. doi:10.1056/NEJM199607113350204

9. Gay F, Engelhardt M, Terpos E, et al. From transplant to novel cellular therapies in multiple myeloma: european Myeloma Network guidelines and future perspectives. Haematologica. 2018;103(2):197. doi:10.3324/haematol.2017.174573

10. Wildes TM, Tuchman SA, Klepin HD, et al. Geriatric assessment in older adults with multiple myeloma. J Am Geriatr Soc. 2019;67 (5):987-991. doi:10.1111/jgs.2019.67.issue-5

11. Auner HW, Szydlo R, Hoek J, et al. Trends in autologous hematopoietic cell transplantation for multiple myeloma in Europe: increased use and improved outcomes in elderly patients in recent years. Bone Marrow Transplant. 2015;50(2):209-215. doi:10.1038/ bmt.2014.255

12. Munshi PN, Hari P, Vesole DH, et al. Breaking the glass ceiling of age in transplant in multiple myeloma. Oral presentation at: American Society of Hematology Annual Meeting; December 9, 2019; Orlando, FL.

13. Blommestein HM, van Beurden-tan CHY, Franken MG, et al. Efficacy of first-line treatments for multiple myeloma patients not eligible for stem cell transplantation: a network meta-analysis. Haematologica. 2019;104(5):1026. doi:10.3324/haematol.2018.206912

14. Rehman W, Arfons LM, Lazarus HM. The rise, fall and subsequent triumph of thalidomide: lessons learned in drug development. Ther Adv Hematol. 2011;2(5):291-308. doi:10.1177/2040620711413165

15. Kotla V, Goel S, Nischal S, et al. Mechanism of action of lenalidomide in hematological malignancies. J Hematol Oncol. 2009;2(1):36. doi:10.1186/1756-8722-2-36

16. Syed YY. Lenalidomide: a review in newly diagnosed multiple myeloma as maintenance therapy after ASCT. Drugs. 2017;77 (13):1473-1480. doi:10.1007/s40265-017-0795-0

17. Palumbo A, Falco P, Corradini P, et al. Melphalan, prednisone, and lenalidomide treatment for newly diagnosed myeloma: a report from the GIMEMA-Italian multiple myeloma network. JCO. 2007;25 (28):4459-4465. doi:10.1200/JCO.2007.12.3463

18. McCarthy PL, Owzar K, Hofmeister CC, et al. Lenalidomide after stem-cell transplantation for multiple myeloma. $N$ Engl J Med. 2012;366(19):1770-1781. doi:10.1056/NEJMoa1114083

19. Attal M, Lauwers-cances V, Marit G, et al. Lenalidomide maintenance after stem-cell transplantation for multiple myeloma. $N$ Engl $J$ Med. 2012;366(19):1782-1791. doi:10.1056/NEJMoa1114138

20. Palumbo A, Hajek R, Delforge M, et al. Continuous lenalidomide treatment for newly diagnosed multiple myeloma. $N$ Engl J Med. 2012;366(19):1759-1769. doi:10.1056/NEJMoa1112704

21. Stewart AK, Jacobus S, Fonseca R, et al. Melphalan, prednisone, and thalidomide vs melphalan, prednisone, and lenalidomide (ECOG E1A06) in untreated multiple myeloma. Blood. 2015;126 (11):1294-1301. doi:10.1182/blood-2014-12-613927

22. Zweegman S, van der Holt B, Mellqvist U-H, et al. Melphalan, prednisone, and lenalidomide versus melphalan, prednisone, and thalidomide in untreated multiple myeloma. Blood. 2016;127 (9):1109-1116. doi:10.1182/blood-2015-11-679415

23. Zonder JA, Crowley J, Hussein MA, et al. Lenalidomide and high-dose dexamethasone compared with dexamethasone as initial therapy for multiple myeloma: a randomized Southwest Oncology Group trial (S0232). Blood. 2010;116(26):5838-5841. doi:10.1182/ blood-2010-08-303487
24. Rajkumar SV, Jacobus S, Callander NS, et al. Lenalidomide plus high-dose dexamethasone versus lenalidomide plus low-dose dexamethasone as initial therapy for newly diagnosed multiple myeloma: an open-label randomised controlled trial. Lancet Oncol. 2010;11 (1):29-37. doi:10.1016/S1470-2045(09)70284-0

25. Benboubker L, Dimopoulos MA, Dispenzieri A, et al. Lenalidomide and dexamethasone in transplant-ineligible patients with myeloma. N Engl J Med. 2014;371(10):906-917. doi:10.1056/NEJMoa1402551

26. Facon T, Dimopoulos MA, Dispenzieri A, et al. Final analysis of survival outcomes in the phase 3 FIRST trial of up-front treatment for multiple myeloma. Blood. 2018;131(3):301-310. doi:10.1182/blood2017-07-795047

27. Magarotto V, Bringhen S, Offidani M, et al. Triplet vs doublet lenalidomide-containing regimens for the treatment of elderly patients with newly diagnosed multiple myeloma. Blood. 2016;127 (9):1102-1108. doi:10.1182/blood-2015-08-662627

28. Larocca A, Salvini M, De Paoli L, et al. Efficacy and feasibility of dose/schedule-adjusted $\mathrm{Rd}-\mathrm{R}$ vs. continuous $\mathrm{Rd}$ in elderly and intermediate-fit newly diagnosed multiple myeloma (NDMM) Patients: RV-MM-PI-0752 Phase III randomized study. Blood. 2018;132(Supplement 1):305. doi:10.1182/blood-2018-99-111796

29. Summary of opinion (post authorisation): Revlimid. European Medicines Agency. Available from: https://www.ema.europa.eu/en/ documents/smop/chmp-post-authorisation-summary-positive-opinion -revlimid en.pdf. Accessed February 17, 2020.

30. Lenalidomide plus dexamethasone for previously untreated multiple myeloma. National Institute for Health and Care Excellence. Available from: https://www.nice.org.uk/guidance/ta587/documents/ final-appraisal-determination-document. Accessed February 17, 2020.

31. Schey S, Montero LFC, Stengel-tosetti C, Gibson CJ, Dhanasiri S. The cost impact of lenalidomide for newly diagnosed multiple myeloma in the EU5. Oncol Ther. 2017;5(1):31-40. doi:10.1007/s40487016-0037-8

32. Quach H, Fernyhough L, Henderson R, et al. Upfront lower dose lenalidomide is less toxic and does not compromise efficacy for vulnerable patients with relapsed refractory multiple myeloma: final analysis of the Phase II RevLite study. Br J Haematol. 2017;177 (3):441-448. doi:10.1111/bjh.2017.177.issue-3

33. Nakaya A, Fujita S, Satake A, et al. Realistic lenalidomide dose adjustment strategy for transplant-ineligible elderly patients with relapsed/refractory multiple myeloma: japanese real-world experience. Acta Haematol. 2017;138(1):55-60. doi:10.1159/ 000477792

34. Durie BGM, Hoering A, Abidi MH, et al. Bortezomib with lenalidomide and dexamethasone versus lenalidomide and dexamethasone alone in patients with newly diagnosed myeloma without intent for immediate autologous stem-cell transplant (SWOG S0777): a randomised, open-label, phase 3 trial. The Lancet. 2017;389 (10068):519-527. doi:10.1016/S0140-6736(16)31594-X

35. O'donnell EK, Laubach JP, Yee AJ, et al. A phase 2 study of modified lenalidomide, bortezomib and dexamethasone in transplant-ineligible multiple myeloma. Br J Haematol. 2018;182 (2):222-230. doi:10.1111/bjh.2018.182.issue-2

36. Facon T, Kumar S, Plesner T, et al. Daratumumab plus lenalidomide and dexamethasone for untreated myeloma. N Engl J Med. 2019;380 (22):2104-2115. doi:10.1056/NEJMoa1817249

37. Zweegman S, Usmani SZ, Chastain K, et al. Bortezomib, lenalidomide, and dexamethasone (VRd) \pm daratumumab (DARA) in patients (pts) with newly diagnosed multiple myeloma (NDMM) for whom transplant is not planned as initial therapy: A multicenter, randomized, phase III study (CEPHEUS). JCO. 2019;37(15_suppl):TPS8056-TPS8056. doi:10.1200/JCO.2019.37.15_suppl.TPS8056CEPHEUS

38. Dimopoulos M, Spencer A, Attal M, et al. Lenalidomide plus dexamethasone for relapsed or refractory multiple myeloma. $N \mathrm{Engl}$ $J$ Med. 2007;357(21):2123-2132. doi:10.1056/NEJMoa070594 
39. Knight R, DeLap RJ, Zeldis JB, et al. Lenalidomide and venous thrombosis in multiple myeloma. $N$ Engl J Med. 2006;354:2079-2080.

40. Rajkumar SV, Blood E. Lenalidomide and venous thrombosis in multiple myeloma. $N$ Engl J Med. 2006;354:2079-2080.

41. National Comprehensive Cancer Network Guidelines. Multiple Myeloma (Version 2.2020). https://www.nccn.org/professionals/physi cian_gls/pdf/myeloma.pdf. Accessed November. 8, 2019.

42. Sanfilippo KM, Luo S, Wang T-F, et al. Predicting venous thromboembolism in multiple myeloma: development and validation of the IMPEDE VTE score. Am J Hematol. 2019;94(11):1176-1184. doi:10.1002/ajh.v94.11

43. Covut F, Ahmed R, Samaras CJ, et al. External validation of the impede VTE risk score in newly diagnosed Multiple Myeloma (MM) patients. Poster presented at: American Society of Hematology Annual Meeting; December 8, 2019; Orlando, FL.

44. Revlimid package insert. Available from: https:/www.accessdata.fda.gov/ drugsatfda_docs/label/2013/021880s034lbl.pdf. Accessed November. 8, 2019

45. Reece D, Kouroukis CT, Leblanc R, et al. Practical approaches to the use of lenalidomide in multiple myeloma: a canadian consensus. $A d v$ Hematol. 2012;2012:621958.

46. Palumbo A, Bringhen S, Kumar SK, et al. Second primary malignancies with lenalidomide therapy for newly diagnosed myeloma: a meta-analysis of individual patient data. Lancet Oncol. 2014;15 (3):333-342. doi:10.1016/S1470-2045(13)70609-0

47. Dimopoulos MA, Richardson PG, Brandenburg N, et al. A review of second primary malignancy in patients with relapsed or refractory multiple myeloma treated with lenalidomide. Blood. 2012;119 (12):2764-2767. doi:10.1182/blood-2011-08-373514

48. Wildes TM, Campagnaro E. Management of multiple myeloma in older adults: gaining ground with geriatric assessment. J Geriatr Oncol. 2017;8(1):1-7. doi:10.1016/j.jgo.2016.04.001

49. Palumbo A, Bringhen S, Mateos M-V, et al. Geriatric assessment predicts survival and toxicities in elderly myeloma patients: an International Myeloma Working Group report. Blood. 2015;125 (13):2068-2074. doi:10.1182/blood-2014-12-615187
50. Engelhardt M, Dold SM, Ihorst G, et al. Geriatric assessment in multiple myeloma patients: validation of the International Myeloma Working Group (IMWG) score and comparison with other common comorbidity scores. Haematologica. 2016;101(9):1110. doi:10.3324/ haematol.2016.148189

51. Facon T, Dimopoulos MA, Meuleman N, et al. A simplified frailty scale predicts outcomes in transplant-ineligible patients with newly diagnosed multiple myeloma treated in the FIRST (MM-020) trial. Leukemia. 2020;34(1):224-233. doi:10.1038/s41375-019-0539-0

52. Cook G, Royle K-L, Pawlyn C, et al. A clinical prediction model for outcome and therapy delivery in transplant-ineligible patients with myeloma (UK myeloma research alliance risk profile): a development and validation study. The Lancet Haematol. 2019;6(3):e154-e166. doi:10.1016/S2352-3026(18)30220-5

53. Nathwani N, Kurtin SE, Lipe B, et al. Integrating touchscreen-based geriatric assessment and frailty screening for adults with multiple myeloma to drive personalized treatment decisions. JOP. 2019: JOP.19.00208. doi:10.1200/JOP.19.00208.

54. Mateos M-V, Dimopoulos MA, Cavo M, et al. Daratumumab plus bortezomib, melphalan, and prednisone for untreated myeloma. $N$ Engl J Med. 2017;378(6):518-528. doi:10.1056/NEJMoa1714678

55. ASCO Post Staff. FDA approves daratumumab/VMP combination for newly diagnosed multiple myeloma. The ASCO Post May 25. 2018. https://www.ascopost.com/issues/may-25-2018/fda-approvesdaratumumab-vmp-combination/. Accessed November 19, 2019.

56. Mateos M-V, Cavo M, Blade J, et al. Overall survival with daratumumab, bortezomib, melphalan, and prednisone in newly diagnosed multiple myeloma (ALCYONE): a randomised, open-label, phase 3 trial. The Lancet. 2020;395(10218):132-141. doi:10.1016/S01406736(19)32956-3

57. Mina R, Bringhen S, Wildes TM, Zweegman S, Rosko AE, Approach to the older adult with multiple myeloma. Am Soc Clin Oncol Educ Book. 2019;39:500-518. doi:10.1200/EDBK_239067
Clinical Interventions in Aging

\section{Publish your work in this journal}

Clinical Interventions in Aging is an international, peer-reviewed journal focusing on evidence-based reports on the value or lack thereof of treatments intended to prevent or delay the onset of maladaptive correlates of aging in human beings. This journal is indexed on PubMed Central, MedLine, CAS, Scopus and the Elsevier
Bibliographic databases. The manuscript management system is completely online and includes a very quick and fair peer-review system, which is all easy to use. Visit http://www.dovepress.com/ testimonials.php to read real quotes from published authors. 